\title{
Bat Swarms Intelligent Optimization Multi-Modulus Algorithm and Influence of Modulation Mode on It
}

\author{
Huapeng Wu a, Yecai Guo b \\ College of Electronic \& Information Engineering, Nanjing University of \\ Information Science \& Technology, Nanjing, 210044, China
}

\begin{abstract}
In order to greatly improve the performance of multi-modulus blind equalization algorithm(MMA) and evaluate the influence of modulation mode on it, MMA based on bat swarm intelligent optimization(BA-MMA) is proposed. This proposed algorithm uses the global optimal position vector of a bat swarm which optimized by bat algorithm(BA) as real and imaginary parts simultaneously of the initial optimal weight vector of the MMA to improve convergence speed and reduce mean square error(MSE). The simulation rests with amplitude phase shift keying(APSK), quadrature amplitude modulation(QAM), and phase shift keying (PSK) validate the performance of the proposed algorithm.

Keywords: Multi-modulus blind equalization algorithm(MMA) ; Bat algorithm(BA) ; Global optimal position ; Optimal weight vector ; Modulation signals
\end{abstract}

\section{Introduction}

Blind equalization algorithm is a kind of adaptive algorithm, which doesn't need to transmit the training sequence, only relies on its own receiving sequence statistical characteristics to adjust the equalizer weight vector, and makes the output sequence similar to the sending sequence[1]. Compared with the constant modulus blind equalization algorithm (CMA), multi-modulus blind equalization algorithm (MMA) has faster convergence speed, smaller steady-state mean square error, and also has the ability to equalize the multi-modulus signal and modify phase rotation[2].

Bat algorithm(BA) is a stochastic global optimization algorithm based on population[3]. The global optimal position vector, which is corresponding to the minimum objective function value, is obtained via changing ultrasonic frequency, frequentness and loudness. Compared with other intelligent algorithms, echo location characteristics of the BAcan make the searching process avoid falling into local searching and improve success rate of searching global optimal position.

In this paper, we combine BA with MMA to propose a multi-modulus blind equalization algorithm based on bat swarms intelligent optimization 
(BA-MMA). The simulation rests with amplitude phase shift keying (APSK), quadrature amplitude modulation(QAM), and phase shift keying(PSK) validate the performance of the proposed algorithm.

\section{Multi-Modulus Blind Equalization Algorithm}

Multi-Modulus Signals(MMSs), whose constellation points are distributed in the circles of different radius, which is called as statistical modulus, can't be effectively equalized by the constant modulus blind equalization algorithm(CMA) because there are many different modulus values. For examples, 32PSK signals have only one modulus, 32APSK have three moduli, and 32QAM have five moduli. In order to equalize higher-order multi- modulus signals, it is necessary that the MMA is employed to equalize the constellation points of the MMSs to different circles corresponding to different modulus values.

The structure of BA-MMA is shown in Fig. 1.

In Fig.1, $\boldsymbol{a}(k)$ is a zero mean independent identically distributed signal vector, $\boldsymbol{c}(k)$ is the channel impulse response, $\boldsymbol{n}(k)$ is additive white Gaussian noise, $\boldsymbol{y}_{R}(k)$ and $\boldsymbol{y}_{I}(k)$ are the real and imaginary parts of input signal $\boldsymbol{y}(k)$, respectively. $\boldsymbol{w}_{R}(k)$ and $\boldsymbol{w}_{I}(k)$ are the real and imaginary parts of weight vector $\boldsymbol{w}(k)$, respectively . $z_{R}(k)$ is the real part of output signal $z(k)$ whereas $z_{I}(k)$ is its imaginary part, and $e_{R}(k)$ and $e_{I}(k)$ are the real and imaginary parts of error function $e(k)$.

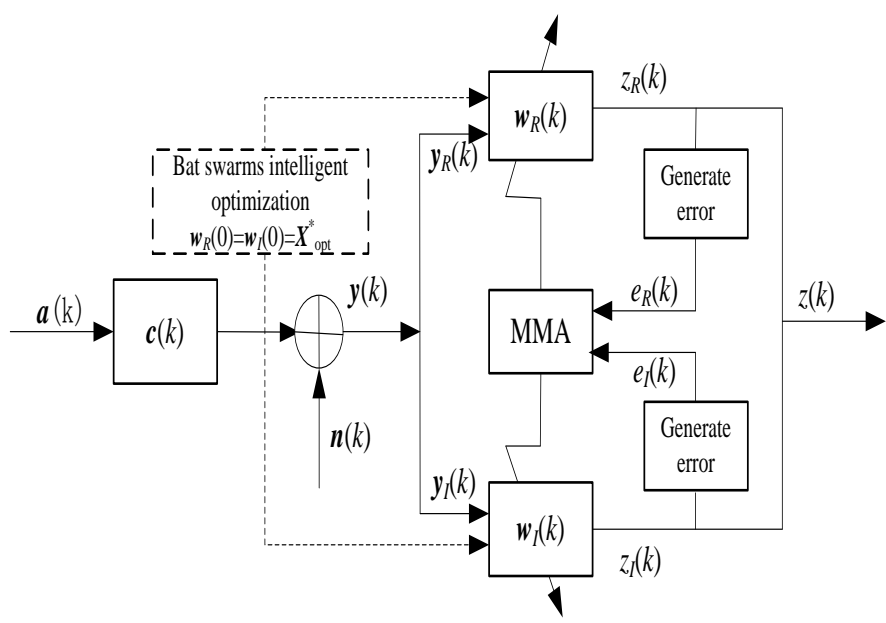

Fig.1 Bat swarms algorithm based MMA

In the MMA, the iterative formulas of $\boldsymbol{w}_{R}(k)$ and $\boldsymbol{w}_{I}(k)$ are defined as

$$
\left\{\begin{array}{l}
\boldsymbol{w}_{R}(k+1)=\boldsymbol{w}_{R}(k)-4 \mu e_{R}(k) \boldsymbol{y}_{R}(k) \\
\boldsymbol{w}_{I}(k+1)=\boldsymbol{w}_{I}(k)-4 \mu e_{I}(k) \boldsymbol{y}_{I}(k)
\end{array}\right.
$$


where $\mu$ is a step-size, $0 \leq \mu \leq 1$.

\section{Multi-Modulus Blind Equalization Algorithm Based on Bat Swarms Intelligent Optimization}

In the bat swarms, the updating equations of positions $\boldsymbol{X}_{i}(k)$ and velocities $\boldsymbol{V}_{i}(k)$ at time $k$ are given as follows

$$
\begin{gathered}
f_{i}=f_{\min }+\left(f_{\max }-f_{\min }\right) \beta \\
(2) \\
\boldsymbol{V}_{i}(k)=\boldsymbol{V}_{i}(k-1)+\left[\boldsymbol{X}_{i}(k)-\boldsymbol{X}^{*}\right] f_{i}
\end{gathered}
$$

$$
\boldsymbol{X}_{i}(k)=\boldsymbol{X}_{i}(k-1)+\boldsymbol{V}_{i}(k)
$$

where $\beta \quad[\nLeftarrow, 1]$ is a random vector which obeys uniform distribution. $f_{\mathrm{i}}$ $\in\left[f_{\min }, f_{\max }\right]$ and is ultrasonic frequency. $\boldsymbol{X}^{*}$ is the current global best position vector.

On the basis of the unique echolocation characteristic of the bat, in the local search procedure, a position vector $\boldsymbol{X}_{\text {old }}$ in all current best position vectors of the bats is selected, the new position vector $\boldsymbol{X}_{\text {new }}$ of all bats is generated by a random walk from $\boldsymbol{X}_{\text {old }}$ and is written as[4]

$$
\boldsymbol{X}_{\text {new }}=\boldsymbol{X}_{\text {old }}+\varepsilon A(k)
$$

where $\varepsilon$ is a random number in [-1,1], $A(k)$ is the average loudness of the whole bat swarm.

After the original position vector $\boldsymbol{X}_{i}(k)$ is replaced with $\boldsymbol{X}_{\text {new }}$, the searching process jumps out of the local searching and returns to global searching to avoid falling into the local optimal.

Bats can emit ultrasonic when they search pray. At first, bats search pray in broader space, the loudness $A$ is large and frequentness $r$ is low. When the bats find their prey, the loudness $A$ can decrease and frequentness $r$ increase in order that bats can search prey more precise. Now we have

$$
\boldsymbol{A}_{i}(k+1)=\alpha \boldsymbol{A}_{i}(k)
$$

$$
r_{i}(k+1)=r(0)[1-\exp (-\gamma k)]
$$

(7)

where $\alpha$ is loudness attenuation coefficient, $\gamma$ is frequentness attenuation coefficient.

In the initialization process, each bat have different frequentness and loudness, the maximum loudness $A(0) \in[1,2]$ and the maximum frequentness $r(0) \in[0,1]$. When best position changes, loudness and frequentness are synchronously 
updated and all bats move towards new best position.

In this paper, the cost function of the MMA is used to define the object function of the proposed BA-MMA. The closer is distance between bat and prey, the greater is the probability that bats catch prey, position corresponding to greater probability is better and the object function value is smaller. Process of searching prey and moving for bats is similar to the good position instead of poor one. Through this searching process, the global optimal location $\boldsymbol{X}_{\mathrm{opt}}{ }^{*}$ can be found and regarded as the original weight vector $\boldsymbol{w}_{R}(0)$ and $\boldsymbol{w}_{I}(0)$ to update the weight vector $\boldsymbol{w}_{R}(k)$ and $\boldsymbol{w}_{I}(k)$ and obtain $\boldsymbol{y}_{R}(k)$ and $\boldsymbol{y}_{I}(k)$, respectively. Object function of thr BA-MMA is defined as

$$
J_{B A-M M A}\left(X_{i}\right)=E\left\{\left[z_{R}^{2}(k)-R_{R}^{2}\right]^{2}+\left[z_{I}^{2}(k)-R_{I}^{2}\right]^{2}\right\}
$$

where $z_{R}(k)=\boldsymbol{X}_{i}(k) \cdot \boldsymbol{y}_{R}(k)$ and $z_{I}(k)=\boldsymbol{X}_{i}(k) \cdot \boldsymbol{y}_{I}(k), \boldsymbol{X}_{i}(k)$ corresponds to the real and imaginary part of a weight vector of MMA.

The implementation processes of BA-MMA are as follows:

Step 1: Parameter initialization. Parameters are given as follows: a random bat swarm, the bat number $n$, frequency range $\left[f_{\min }, f_{\max }\right]$, maximum loudness $A(0)$, maximum frequentness $r(0)$, searching accuracy tol, loudness attenuation coefficient $\alpha$, frequentness attenuation coefficient $\gamma$, dimension number $d$, maximum iteration number iter, running number runs, channel $\boldsymbol{h}$, signal to noise ratio(SNR) snr, the number of equalizer taps $L$, and position vector of each bat $\boldsymbol{X}_{i}$.

Step 2: Calculate object function value. The object function value is calculated according to Eq.8, the position corresponding to the minimum object function is called as the current global optimal position $\boldsymbol{X}^{*}$.

Step 3: Adjust frequency $f_{\mathrm{i}}$ and update position and velocity of each bat according to Eq.3 and Eq.4 to achieve the updated position $\boldsymbol{X}_{i}(k)$ [5].

Step 4: Generate a random rand1 and compare with frequentness $r_{\mathrm{i}}$. If rand $1>r_{\mathrm{i}}$, a new position is generated by a random walk from the current optimal position and the local searching process returns to global searching.

Step 5: Generate a random rand2 and compare with loudness $A_{i}$. If rand2>Ai and $J_{\text {BA-MMA }}\left(\boldsymbol{X}_{i}(k)\right)<J_{\text {BA-MMA }}\left(\boldsymbol{X}^{*}\right)$, the current global optimal position $\boldsymbol{X}^{*}$ is replaced with the current position vector $\boldsymbol{X}_{i}$ and $A_{i}$ and $r_{\mathrm{i}}$ are updated according to Eq.6 and Eq.7.

Step 6: Choose the current global optimal position $\boldsymbol{X}^{*}$.

Step 7: Obtain the global optimal position vector. When the searching process reaches to the maximum iteration number or the searching accuracy, the global optimal position $\boldsymbol{X}^{*}$ is written as $\boldsymbol{X}_{\mathrm{opt}}{ }^{*}$. Otherwise, the searching process turns to step 3.

Step 8: Obtain the original optimal position vector. The global optimal position $\boldsymbol{X}_{\text {opt }}{ }^{*}$ is regarded as the real and imaginary parts of the weight vector, meanwhile. That is, $\boldsymbol{w}_{R}(0)=\boldsymbol{X}_{\mathrm{opt}}{ }^{*}, \boldsymbol{w}_{I}(0)=\boldsymbol{X}_{\mathrm{opt}}{ }^{*}, \boldsymbol{w}_{R}(k)$ and $\boldsymbol{w}_{I}(k)$ can be updated according to Eq.1 and Eq.2, respectively, then $\boldsymbol{y}(k)$ can be equalized more effectively. 


\section{Test results}

In order to verify the performance of BA-MMA and the influence of the modulation mode on it, simulation experiments with different modulation signals are carried out.

Initialization of parameters: $n=20, f_{\mathrm{i}} \in[0,100], A(0)=1.5, r(0)=0.25$, tol $=10^{-5}, d=11, \alpha=0.9, \gamma=0.9$, iter $=2000$, runs $=2000, \boldsymbol{h}=[0.9556$ -0.09060 .05780 .2368 ], snr=25dB, $L=11$.

In the first simulation, the transmitted signal are 16APSK, 16QAM and 16PSK, the step-size is 0.002, the simulation results are shown in Fig.2. In the second simulation, we use 32APSK,32QAM and 32PSK signals for the transmitted signal, the step-size is 0.001 , Fig. 3 is simulation results .

Fig. 2 and Fig.3 show that BA-MMA has huge advantages in equalizing high order multi- modulus signals, improving the convergence speed and reducing MSE. But because of different modulation modes, equalization effects are also different. From Fig.2 and Fig.3, we can also know that BA-MMA has the best equalization effect for QAM signals, both 16QAM and 32QAM have the minimum MSE and the clearest and most compact Constellations.
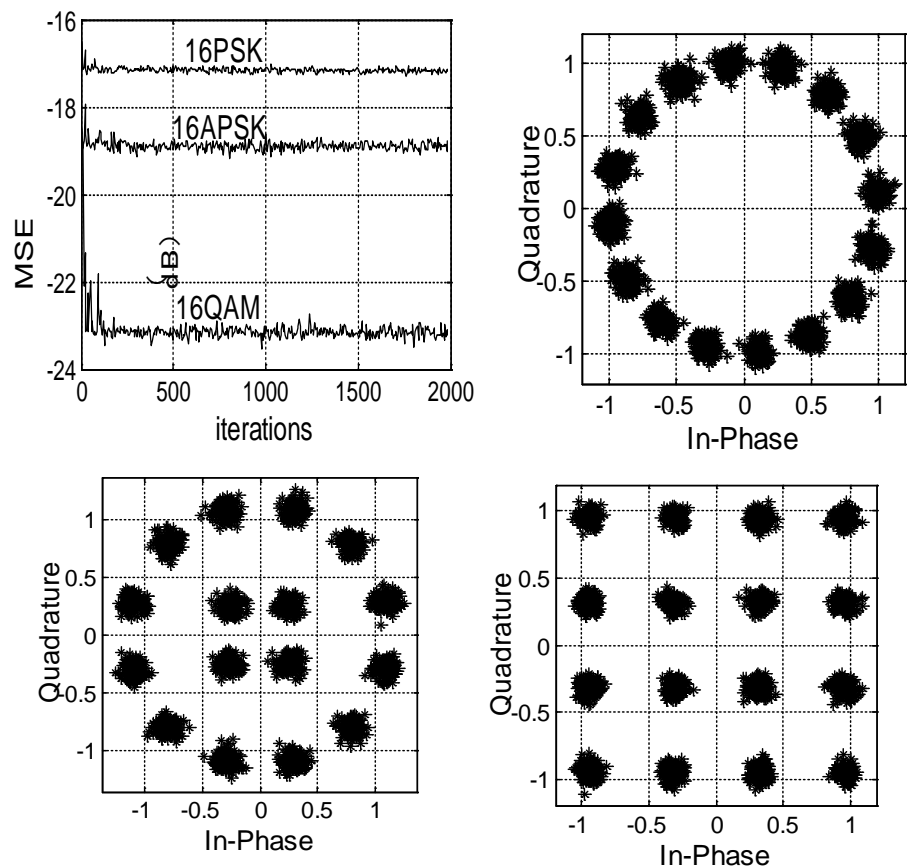

(a) Mean square error

(b) 16PSK signals

(c) 16APSK signals

(d)

Fig.2 Simulation results of 16APSK,16QAM, and 16PSK modulation signals 

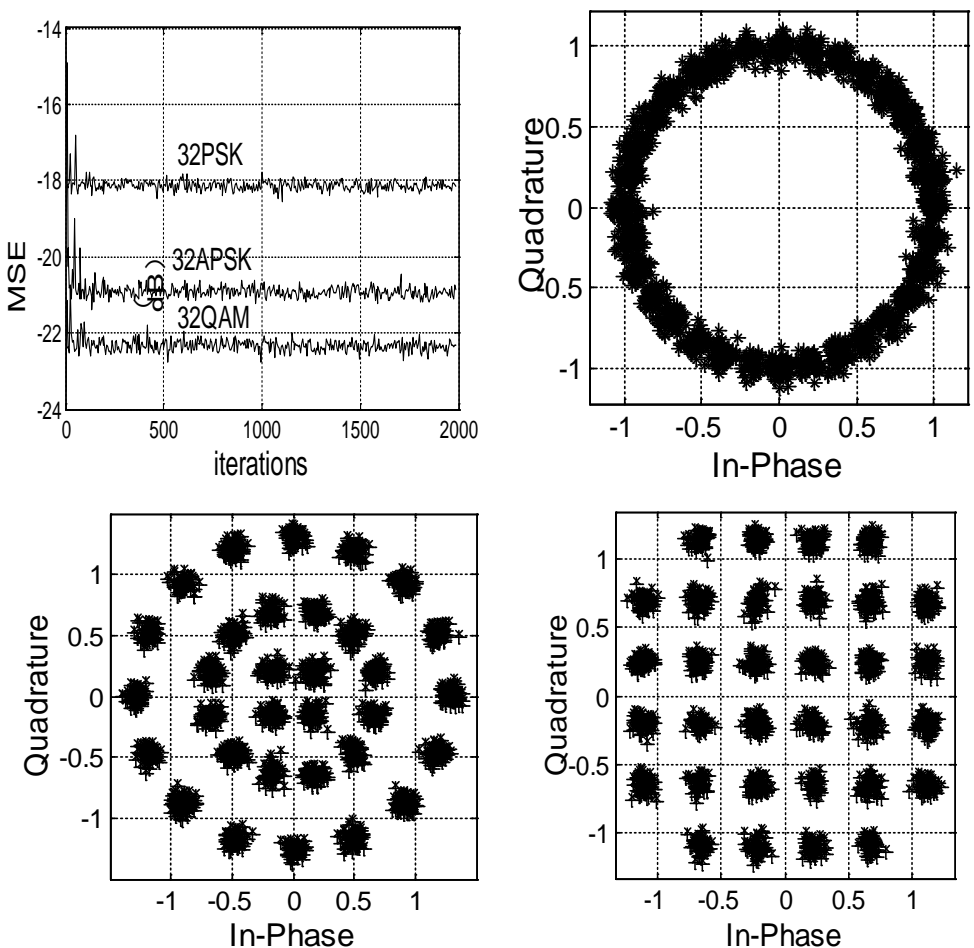

(a) Mean square error

(b) 32PSK signals 32QAM signals

(c) 32APSK signals

(d)

Fig.3 Simulation results of 32APSK,32QAM,and 32PSK modulation signals

\section{Conclusion}

A multi-modulus blind equalization algorithm based on bat swarm intelligent optimization (BA- MMA) is proposed in this paper. Theoretical analysis and simulation results show that, the proposed algorithm has excellent effect on equalizing high order multi-modulus signals, and the equalization effect is affected by modulation.

\section{Acknowledgement}

This work is supported by the major project of University Natural Science of Jiangsu Province (No.13KJA510001), Jiangsu Scientific Research Achievements in Industrialization Project (JHB 2012-9), and A Project Funded by the Priority Academic Program Development of Jiangsu Higher Education Institutions(PAPD II), as well as Innovation Project of Postgraduate Training in Jiangsu Province(No. SJZZ_0110, SJZZ_0111, and KYLX_0853). 


\section{References}

[1] W.Siyuan, L.Feng: A Computationally Efficient Multi-Modulus Blind Equalization Algorithm. Information Management and Engineering(ICIME)(2010)

[2] Y.Jenqtay, C.Jenhung and L.Tzuchao: Effect of Channel Noise on Blind Equalization and Carrier phase Recovery of CMA and MMA. IEEE. Vol. 60(2012), p. 3274

[3] Y.Jian, J.J.Werner and G.A.Dumont: The Multimodulus Blind Equalization and Its Generalized Algorithms. IEEE . Vol. 20(2002), p. 997

[4] Y.Xinshe, in: Nature Inspired Cooperative Strategies for Optimization (NISCO), edited by J.R.Gonzalez, Springer, Berlin(2010)

[5] Y.Xinshe, M.Karamanoglu and S.Fong: Bat Algorithm for Topology Optimization in Microelectronic Applications. Future Generation Communication Technology(FGCT)(2012) 